\title{
Epidemiologic Profile of Patients with Snoring and Obstructive Sleep Apnea in a University Hospital
}

\author{
Felipe Almeida Mendes ${ }^{1}$ Silvio Antonio Monteiro Marone ${ }^{1}$ Bruno Bernardo Duarte ${ }^{1}$ \\ Ana Carolina Parsekian Arenas ${ }^{1}$
}

${ }^{1}$ Department of Otolaryngology, Pontifícia Universidade Católica de Campinas PUC-Campinas, Campinas, SP, Brazil

Address for correspondence Felipe Almeida Mendes, Medical Doctor Specialist in Otorhinolaryngology by the Brazilian Association of Otolaryngology and Head and Neck Surgery, Department of Int Arch Otorhinolaryngol 2014;18:142-145. Otolaryngology, Pontifícia Universidade Católica de Campinas PUCCampinas, Av. John Boyd Dunlop Jardim Ipaussurama, Campinas, São Paulo 13060-904, Brazil (e-mail: felipemendes@hotmail.com).

\begin{abstract}
Introduction There are several studies on the pathophysiology and prevalence of Obstructive Sleep Apnea Syndrome (OSAS), however, few studies address the epidemiological profile of these patients.

Objective The aim of this study is to analyze the epidemiological profile of patients diagnosed with OSAS referred to the Sleep Medicine clinic.

Methods Cross-sectional individualized study covering 57 patients who were referred from the general ENT clinic to the Sleep Medicine clinic.

Results Classification of OSAS: $16 \%$ had primary snoring, $14 \%$ mild OSAS, $18 \%$ moderate OSAS, and 52\% severe OSAS. Distribution according to weight: $7 \%$ had normal weight, $2 \%$ were overweight (BMI 2530), 37\% grade I obesity (BMI 25.1 to 30); $9 \%$ grade II obesity (BMI 30.1 to 35 ) and grade III obesity (BMI greater than 35 ) in $45 \%$ of cases. Distribution Friedmann stage: $9 \%$ were classified as grade I, $35 \%$ were considered grade II, $54 \%$ as grade III and $2 \%$ as grade IV. Treatment adopted: $46 \%$ were treated with CPAP; $19 \%$ were treated with surgery; oral appliance was designed for $14 \%$ patients, $7 \%$ were given roncoplastic injection and 7\% positional therapy. A new polysomnography was asked to $5 \%$ of patients. To $2 \%$ of patients given the oral appliance was due to

Keywords

- snoring

- sleep apnea

- obstructive

- health profile treatment failure with roncoplastic injection.

Conclusion Most of the patients are male, obese and with moderate or severe OSAS. Snoring and daytime excessive sleepiness were the most common symptoms. The surgical procedures employed in this service (roncoplastic injection, UPPP and lateral pharyngoplasty) followed the recommendations of the available literature.
\end{abstract}

\section{Introduction}

Obstructive sleep apnea syndrome (OSAS) is characterized by episodes of obstruction (partial or total) of the upper airway during sleep secondary to a collapse of the structures of the upper airway during inspiration. OSAS manifests itself as a reduction (hypopnea) or complete cessation (apnea) of airflow despite continued respiratory efforts. OSAS is diagnosed by clinical history and polysomnography. OSAS is defined by an apnea-hypopnea index (AHI) $>15$ or an AHI $>5$ with daytime and nighttime symptoms. The apnea severity is classified as mild (AHI 5 to 15), moderate (AHI 15.01 to 30), or severe (AHI > 30.1).

One of the most common clinical signs of sleep apnea is snoring. It is estimated that $~ 45 \%$ of men and $30 \%$ of women received

August 27, 2013

accepted

August 29, 2013
DOI http://dx.doi.org/

10.1055/s-0033-1359309. ISSN $1809-9777$.
Copyright $\odot 2014$ by Thieme Publicações License terms Ltda, Rio de Janeiro, Brazil

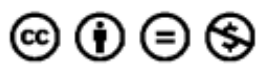


over 65 snore. Other usual symptoms associated with OSAS are excessive daytime sleepiness, nocturnal awakenings, fatigue, and headache upon waking in the morning. ${ }^{1}$

A lack of adequate ventilation results in an oxyhemoglobin desaturation and, in severe cases, hypercapnia. OSAS is associated with a variety of pathophysiological changes that impair cardiovascular function including increased inflammatory markers and blood pressure peaks during sleep. There is increasing evidence that OSAS increases the incidence of hypertension, stroke, myocardial infarction, and premature death. ${ }^{2,3}$

The prevalence of OSAS varies depending on the population studied and the diagnostic criteria used. Young et $\mathrm{al}^{4}$ found that population prevalence was $9 \%$ in women and $24 \%$ men (using as criterion only AHI $>5$ ) in subjects aged 30 to 60 years. However, the prevalence drops to $4 \%$ in men and $2 \%$ in women when taking into account the complaint of excessive daytime sleepiness with $\mathrm{AHI}>5$.

Another study by Young and colleagues ${ }^{5} 5$ estimated that the prevalence of mild OSAS may vary from 3 to $28 \%$ of the adult population, whereas moderate to severe OSAS (AHI $\geq$ 15) may range from 1 to 14\%. Importantly, the authors argued that OSAS is underdiagnosed in a high percentage of cases in which there is no complaint of excessive daytime sleepiness. This study also demonstrated that OSAS is more prevalent in men than women (ratio of 2 to $3: 1$ ), that there is an increased prevalence among the elderly (especially those over 65 years), and that pregnancy is a risk factor for OSAS.

Bixler et $\mathrm{al}^{6}$ found that the prevalence of OSAS was 3.9\% among men and $1.2 \%$ among women (3.3:1) considering an $\mathrm{AHI} \geq 10$. The prevalence in women before menopause (or use of hormone replacement therapy) was 0.6 versus $2.7 \%$ in women after menopause (and without hormone replacement therapy).

Clearly other factors that increase the risk for OSAS, including age, structural abnormalities in the upper airway, use of sedatives and alcohol, and probably familial history. ${ }^{1}$ It is also the consensus that the prevalence of OSAS increases in the obese population (BMI $>30$ ). In a study published in Japan $^{7}$ involving 275 men, the prevalence of severe OSAS (AHI > 30) was 1 in 6 individuals with metabolic syndrome (BMI > 30 associated with dyslipidemia and/or hyperglycemia), whereas the prevalence was only 1 in 40 individuals without the metabolic syndrome.

Tufik et $\mathrm{al}^{8}$ in his study of 1,042 volunteers undergoing overnight polysomnography showed that $32.8 \%$ of residents of São Paulo presented the criteria for OSAS according to the American Academy of Sleep Medicine (AHI $>15$ or AHI $>5$ with daytime and nighttime symptoms). Prevalence estimates are higher among men and increase in both sexes with age. OSAS was also more prevalent in overweight and obese men and women. This same study showed that $55 \%$ of the population suffer from drowsiness, $38.9 \%$ from fatigue, and $20.5 \%$ reported snoring. AHI below 5 was present in $61.8 \%$ of patients, $21.3 \%$ presented with AIH between 5 to 14.9 and $16.9 \%$ had an $\mathrm{AHI} \geq 15$. The $\mathrm{AHI} \geq 15$ was more common among older individuals and men.
Overweight and obese men and women were also more likely to have an AHI > 15, and men with high socioeconomic status and women are less likely to have a $\mathrm{AIH}>15$.

These variations are partly a result of the lack of homogeneity in epidemiologic studies. Some studies, for instance, were conducted in groups of preselected populations (e.g., industrial workers or clinically referred patients) and included a large number of individuals with suspected OSAS due to the frequency of snoring. Also, some previous studies did not include patients over 60 years of age.

The vast majority of OSAS studies are dedicated to assessing the prevalence of OSAS in the population, and this has great value for understanding the disease. The aim of this study is to analyze the epidemiologic profile of patients referred to the sleep medicine clinic who were previously selected in the otorhinolaryngology ward of a university hospital, as well as the treatment determined for each patient.

\section{Materials and Methods}

This is a cross-sectional and individualized study covering 57 patients who were referred from the general ear, nose, and throat (ENT) clinic to the sleep medicine clinic between April 2007 and January 2012. All patients signed an informed consent and the study was approved by the ethics committee (protocol number 0807/11).

All patients came to the clinic with their respective roommates and their polysomnography report, were questioned about the possible symptoms, and had a complete ENT examination. In addition, patients completed the Epworth scale of daytime sleepiness and a specific protocol for patients with snoring and sleep apnea.

\section{Results}

It was found that $68 \%$ of patients were men $(n=39)$ and $32 \%$ were women $(n=18)$. The higher incidence in males is consistent with the literature ${ }^{1-8}$ (-Fig. 1). The average age of participants was 47.86 years.

Regarding the classification of OSAS (-Fig. 2), $16 \%$ of patients had primary snoring, $14 \%$ mild OSAS, $18 \%$ moderate OSAS, and 52\% severe OSAS. The highest prevalence of moderate and severe OSAS can be attributed to the fact that these patients tend to be more symptomatic, so they are more likely to seek medical help. The higher incidence of moderate and severe OSAS explains the high incidence of symptoms indicative of impaired sleep quality and excessive daytime sleepiness (reported in 69\% of patients), nighttime awakenings ( $66 \%$ of patients), and morning headache ( $49 \%$ of

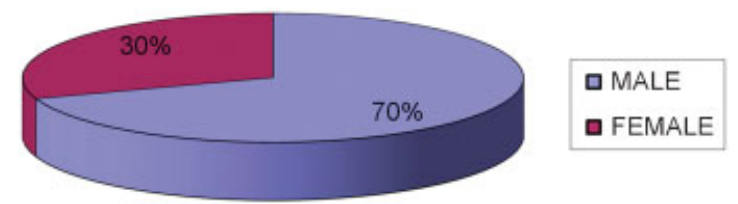

Fig. 1 Distribution according to gender. 


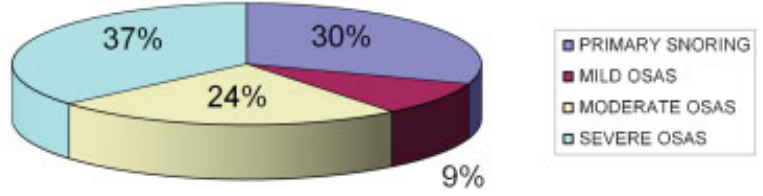

Fig. 2 Distribution according to the severity of obstructive sleep apnea syndrome (OSAS).

patients). Snoring was reported by $90 \%$ of patients, the most prevalent symptom.

Only $7 \%$ of patients had normal weight (BMI 18 to 25 ), $2 \%$ were overweight (BMI 25 to 30), 37\% had grade I obesity (BMI 25.1 to 30 ), $9 \%$ grade II obesity (BMI 30.1 to 35 ), and $45 \%$ grade III obesity (BMI > 35; ( - Fig. 3 ).

According to Friedmann stage ( - Fig. 4), only 9\% of the patients were classified as grade I, $35 \%$ were considered grade II, $54 \%$ as grade III, and $2 \%$ as grade IV.

In this study, we analyzed the treatment adopted in each case (-Fig. 5). The majority of patients (46\%) were treated with continuous positive airway pressure (CPAP). Surgery (uvulopalatopharyngoplasty [UPPP] or lateral pharyngoplasty) was given to $19 \%$ of patients, the mandibular advancement oral applience was designed for $14 \%$ patients, $7 \%$ were given roncoplastic injection, and 7\% received positional therapy. A new type of polysomnography was administered to $5 \%$ of patients. The oral appliance was given to $2 \%$ of the patients because of roncoplastic injection treatment failure.

\section{Discussion}

Sleep medicine is a new science, and knowledge has evolved exponentially in recent years. Within this concept, the study of OSAS, the most common sleep disturbance in the general population including all age groups, is of utmost importance. Therefore, knowing that most patients are undiagnosed, we decided to determine and analyze the epidemiologic profile of patients with snoring and sleep apnea in the database of a university hospital.

When analyzing the sex distribution of patients studied, there is a predominance of males, with a similar distribution to most studies $(2: 1){ }^{3}$ Snoring was the most common patient complaint (90\%), which corroborates the need to consider it as a factor for investigating respiratory sleep disorders. ${ }^{8}$

Excessive daytime sleepiness, unrefreshing sleep, fatigue on waking, morning headaches, and irritability were marked-

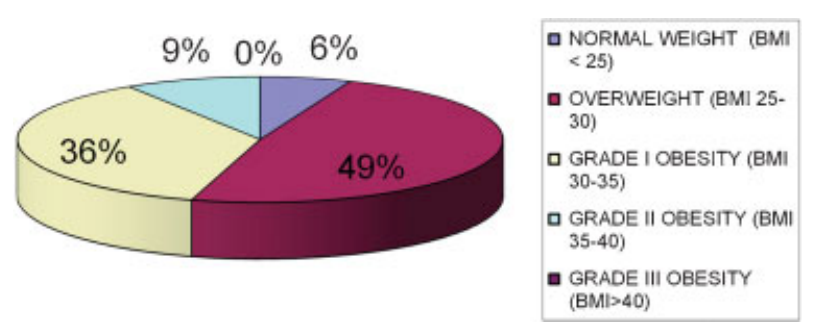

Fig. 3 Distribution according to body mass index (BMI).

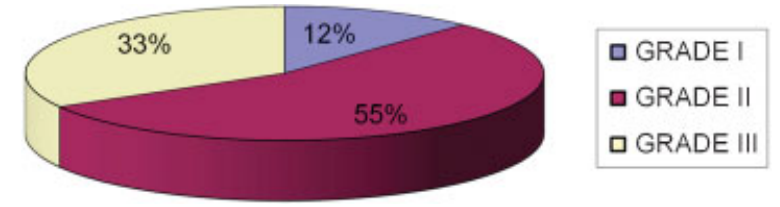

Fig. 4 Distribution according to the Friedmann stage.

ly common in our patients, confirming the significant interference of OSAS on quality of life, social relationships, family, labor, and the risk of accidents. ${ }^{1}$

These data alert health professionals to intensify efforts in the diagnosis and treatment of this important disease. Taking into account the classification of severity of OSAS, we found the following distribution: $16 \%$ primary snoring, $14 \%$ mild OSAS, $18 \%$ moderate OSAS, and 52\% severe OSAS. Moderate and severe OSAS makes up 70\% of this population, an alarming percentage considering the pronounced proven mortality in patients with moderate and severe OSAS. ${ }^{2}$ The high prevalence of moderate and severe cases may be due to the fact that these patients are more symptomatic, and therefore seek medical attention more often.

A minority of patients ( $7 \%$ ) were within the normal BMI (BMI $<25$ ), which highlights the association between obesity and severe OSAS. ${ }^{7,8}$

We found that most patients (46\%) were treated with CPAP, which is considered the gold standard for treatment of OSAS. $^{4,5}$ Intraoral device was indicated for $14 \%$ of patients and only $19 \%$ of patients were treated surgically; of these, $64 \%$ underwent the lateral pharyngoplasty.

There were no indications of any surgical procedure in patients with a BMI $>35$ (obesity class II or III) due to success rates in this group decreasing mainly due to a sharp narrowing pharyngeal and fat accumulation in pharyngeal tissues. However, $93 \%$ of patients included in this study were overweight or obese, indicating a high incidence of OSAS in this group people. ${ }^{7,8}$

In the group of patients undergoing roncoplastic injection, $2(40 \%)$ had mild OSAS and $3(60 \%)$ had primary snoring, which is consistent with the indication in the literature for palatal procedures for the treatment of this pathology. No patient in this group was classified as grade I Friedmann, which would indicate UPPP. ${ }^{9-12}$

Roncoplastic injection is a procedure with a mechanism of action similar to palatal implants and radiofrequency

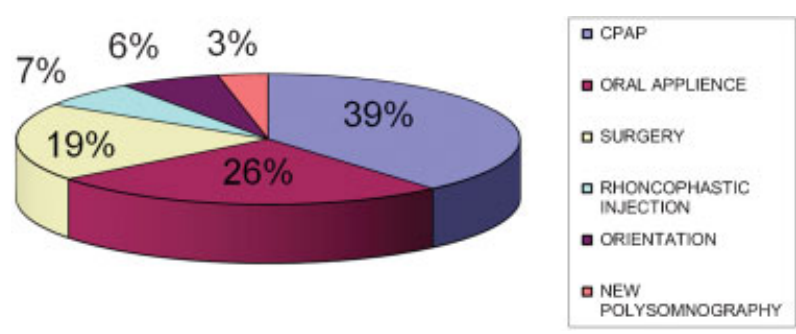

Fig. 5 Treatment. Abbreviation: CPAP, continuous positive airway pressure. 
ablation, which cause sclerosis (hardening) of the soft palate, which prevents vibration. ${ }^{11,12}$

In the group of patients who underwent UPPP, none were classified as grade III Friedmann (due to low success rates in this group). This procedure was performed in five patients, two of whom were classified as grade I and two as grade II. In this group of patients, two had severe OSAS, one had moderate OSAS, one had mild OSAS, and one had primary snoring. This heterogeneous distribution shows that the AHI is less important than the Friedmann classification to indicate a UPPP. $^{9-12}$

Patients classified as Friedmann II who underwent UPPP also underwent nasal surgery (septoplasty and turbinectomy) and suffered from primary snoring or mild OSAS. The main complaint of these patients regarding sleeping was social embarrassment caused by snoring, and none reported excessive daytime sleepiness or fatigue on awakening. Nasal obstructions did not influence the pathophysiology of OSAS, and there was no contraindication to nasal and pharyngeal procedures being performed at the same time. ${ }^{8-10}$

Regarding the lateral pharyngoplasty, patients who underwent this procedure had higher AHI and were classified as having moderate or severe OSAS. In this group of six patients, four had severe OSAS and two had moderate OSAS. Regarding the Friedmann classification, only one patient in this group was classified as grade I. The lateral pharyngoplasty surgical option was used when the patient had moderate or severe OSAS, bulky rear pillar, or larynx position cranial to nasofibrolaryngoscopy and when the anatomy was not favorable for the realization of UPPP. ${ }^{10-12}$

The maxillomandibular advancement was not performed because there was no formal indication. ${ }^{10}$

\section{Conclusion}

From the data analyzed in this study, we conclude that the epidemiologic profile of patients seen at the sleep medicine clinic in a university hospital are mostly obese menwith moderate or severe OSAS. Snoring and daytime excessive sleepiness were the most common symptoms. The surgical procedures employed in this service (roncoplastic injection, UPPP, and lateral pharyngoplasty) were given following the recommendations of the available literature. We also noted the reduced indication of surgical treatment, especially UPPP, which is directly related to the fact that most patients were not classified as grade I in the Friedmann scale.

\section{References}

1 Bresnitz EA, Goldberg R, Kosinski RM. Epidemiology of obstructive sleep apnea. Epidemiol Rev 1994;16(2):210-227

2 Marshall NS, Wong KKH, Liu PY, Cullen SRJ, Knuiman MW, Grunstein RR. Sleep apnea as an independent risk factor for allcause mortality: the Busselton Health Study. Sleep 2008;31(8): 1079-1085

3 Durán J, Esnaola S, Rubio R, Iztueta A. Obstructive sleep apneahypopnea and related clinical features in a population-based sample of subjects aged 30 to $70 \mathrm{yr}$. Am J Respir Crit Care Med 2001;163(3 Pt 1):685-689

4 Young T, Palta M, Dempsey J, Skatrud J, Weber S, Badr S. The occurrence of sleep-disordered breathing among middle-aged adults. N Engl J Med 1993;328(17):1230-1235

5 Young T, Peppard PE, Gottlieb DJ. Epidemiology of obstructive sleep apnea: a population health perspective. Am J Respir Crit Care Med 2002;165(9):1217-1239

6 Bixler EO, Vgontzas AN, Lin HM, et al. Prevalence of sleep-disordered breathing in women: effects of gender. Am J Respir Crit Care Med 2001;163(3 Pt 1):608-613

7 Chin K, Oga T, Takahashi K, et al. Associations between obstructive sleep apnea, metabolic syndrome, and sleep duration, as measured with an actigraph, in an urban male working population in Japan. Sleep 2010;33(1):89-95

8 Tufik S, Santos-Silva R, Taddei JA, Bittencourt LR. Obstructive sleep apnea syndrome in the Sao Paulo Epidemiologic Sleep Study. Sleep Med 2010;11(5):441-446

9 Caples SM, Rowley JA, Prinsell JR, et al. Surgical modifications of the upper airway for obstructive sleep apnea in adults: a systematic review and meta-analysis. Sleep 2010;33(10): 1396-1407

10 Aurora RN, Casey KR, Kristo D, et al; American Academy of Sleep Medicine. Practice parameters for the surgical modifications of the upper airway for obstructive sleep apnea in adults. Sleep 2010; 33(10):1408-1413

11 Cahali MB. Lateral pharyngoplasty: a new treatment for obstructive sleep apnea hypopnea syndrome. Laryngoscope 2003; 113(11):1961-1968

12 Lorenzetti FT, Formigoni GG, Cahali MB. A proposition for a new term: "Rhonchoplastic injection.". Braz J Otorhinolaryngol 2008; 74(3):327 